\title{
Local Differential Excitation Binary Co-occurrence Pattern (LDEBCoP): A New Descriptor for Texture and Bio-Medical Image Retrieval
}

\author{
G V Satya Kumar ${ }^{1}$, P G Krishna Mohan ${ }^{2}$ \\ ${ }^{I}$ Research scholar, Dept. of ECE, JNT University, Hyderabad, India \\ ${ }^{2}$ Professor, Dept. of ECE, IARE, JNT University, Hyderabad, India
}

\begin{abstract}
This paper presents a novel pattern based feature descriptor named as Local Differential Excitation Binary Co- occurrence Pattern (LDEBCoP) for texture and biomedical image retrieval. The proposed method exploits the local structure information using differential excitation. Further, to produce more compact local binary patterns the adjacent neighbourhood pixel pairs are considered in the computation of differential excitation. In the proposed method, the co-occurrence of pixel pairs in local binary map have been observed using gray level co-occurrence matrix(GLCM) in different directions and distances for better feature representation. Previous methods have utilized histogram to obtain the frequency information of local pattern map but co- occurrence of pixel pairs is more robust than frequency of patterns. The performance of proposed method is compared with the state of the art pattern based techniques on the results obtained using various bench mark image databases viz., KTH-TIPS, OUTEX texture database, NEMA-CT database and VIA/IELCAP database which also includes region of interest CT images.
\end{abstract}

Index Terms: Differential excitation, Local binary pattern, Image retrieval, GLCM, Pattern recognition.

\section{Introduction}

Image analysis through local patterns play a vital role in many machine vision applications including computer assisted diagnosis, texture recognition, object recognition and tracking, remote sensing, face recognition and crowd monitoring etc[1,2]. The main advantage of pattern based methods is that they do not require segmentation and are very distinctive and robust to occlusion. A good local feature descriptor can tolerate illumination changes, image compression, image blur and small perspective distortions while preserving distinctiveness.

Local binary patterns(LBP) gained their popularity because of their ability to code fine details, resistance to light changes and low computational complexity [3]. The LBP and their extensions are computationally efficient due to their unified approach of statistical and structural characteristics to describe features. The adoption of local thresholding and histogram representation to describe features make LBP and their extensions more computationally efficient.

The ability to represent textures of different granularities and robustness to illumination variations make LBP based descriptors suitable for applications like face recognition, gender classification and many more industrial applications [4-7]. Many enhancements have been derived to the basic LBP proposed by Ojala et al [8]. Various LBP based descriptors and their applications in computer vision are presented by Zhao et al [9]. The illumination variance and rotation invariance have been widely investigated in [10-13].

Tan and Triggs [14] devised Local Ternary Pattern (LTP) as an extension to basic LBP. LTP can be decomposed into two local binary patterns and LTP is less sensitive to noise in uniform regions. However, the feature vector length is twice that of LBP. Later, Mäenpää. T et al [15] extended applications of LBP to color image analysis. Apart from texture analysis, the pattern based techniques also find application in bio-medical image retrieval.

With advancement in medical technology, there has been an exponential growth in medical images in hospitals and medical institutions in order to meet modern medical requirements. The medical images are stored in different formats such as Computer Tomography (CT), Ultrasound, X-ray images, Magnetic Resonance Images (MRI) etc. Managing and archiving this huge data by human annotation is a herculean task triggering a desperate need for efficient image indexing and retrieval techniques like content based image retrieval (CBIR).

Manjunath et al [16] devised a technique based on bit plane histogram and hierarchical bit plane histogram along with Cumulative Distribution Function (CDF) for CT and MRI image retrieval. Woo et al [17] proposed an image retrieval technique for blood cell images, using color histogram and wavelet transform. The classification of different breast cancer masses based on shape and texture features in sonography images was discussed by Zakeri et al [18]. In [19] wavelet transform based brain image retrieval is presented. Medical CT 
and MRI image retrieval using co-occurrence matrix is presented by Felipe et al [20]. Further, image retrieval of different body parts using color quantization and wavelet transform is presented in [21].

Murala et al [22] devised a technique based on binary wavelet and local binary patterns entitled as directional binary wavelet pattern for face and bio-medical image retrieval . Later, Murala et al [23] proposed a novel image retrieval technique for bio-medical image retrieval and indexing called as Local Mesh Pattern (LMeP). Zhang et al [24] presented image retrieval technique based on derivative patterns of LBP called local derivative patterns (LDP). Co-occurrence of pixel pairs in binary pattern map extracted using center symmetric local binary patterns (CSLBP) called center symmetric local binary co-occurrence pattern (CSLBCoP) for texture, face and bio-medical image retrieval is presented in [25]. Local ternary co-occurrence pattern for MRI and CT image retrieval is presented in [26].

The lion's share of the local pattern based techniques relies on gray level intensity difference to extract the local structure information and they ignore the original stimulus intensity. The proposed method derived its motivation from the fact that the human perception of pattern not only depends on the gray level intensity difference but also on the original intensity stimulus. The differential excitation, which is a ratio of difference in gray level intensity to the original stimulus intensity, is used as a feature descriptor along with the most popular local binary pattern for better feature extraction. Further, the differential excitation is computed using the adjacent neighbour pixel pairs to produce compact binary pattern descriptor. The rest of the paper is systematized as follows. Section-II briefly reviews the related work on local pattern based techniques LBP, CSLBP and differential excitation respectively. Section-III presents the concept of proposed system frame work, feature extraction and evaluation measures. Section-IV reports the experimental results on bench mark databases and finally, the paper is concluded in Section-V.

\section{A. Local binary patterns (LBP)}

\section{Related work}

Ojala et al [8] devised local binary patterns (LBP) for texture classification. Later, face recognition, palm print recognition and image retrieval applications were developed using LBP. It achieved success due to its computational efficiency and speed. For a given image, the LBP operator is computed at each pixel location considering a small circular neighbourhood with radius $\mathrm{R}$ and $\mathrm{N}$ pixels around the reference pixel or center pixel as follows

$$
L B P_{N, R}=\sum_{n=0}^{N-1} S\left(Y_{n}-Y_{c}\right) \times 2^{n}
$$

Where $N$ is number of pixels in the neighbourhood, $Y_{n}$ is neighbourhood pixel and $Y_{c}$ is center pixel or reference pixel.

$$
S(x)=\left\{\begin{array}{c}
1, x \geq 0 \\
0, x<0
\end{array}\right.
$$

The histogram of these binary map values is then used to characterize the texture of the image.

\section{B. Center symmetric local binary patterns (CSLBP)}

Heikkilä et al [27] introduced a modified form of local binary pattern called center symmetric local binary pattern (CSLBP). CSLBP exploits the local pattern by considering gray level intensity of center symmetric pixels. Mathematically, CSLBP is explained as follows

$$
\begin{gathered}
C S L B P_{P, R, T}=\sum_{k=0}^{P-1} 2^{k} \times S\left(Y_{c}-Y_{k+P / 2}\right) \\
S(x)= \begin{cases}1 & x>0 \\
0 & \text { else }\end{cases}
\end{gathered}
$$

\section{Differential excitation ( $\xi$ )}

Chen et al [28] introduced the concept of differential excitation. The differential excitation is defined as the ratio of two terms: The gray level difference of center pixel with its neighbours to the center pixel value. Mathematically, differential excitation is computed as follows

$$
\xi=\arctan \left[\sum_{k=0}^{P-1} \frac{\left(Y_{c}-Y_{k}\right)}{Y_{c}}\right]
$$

Where $Y_{c}$ is center pixel, $Y_{k}$ - neighbour pixel. More details about differential excitation can be found in [31]. 


\section{Proposed work}

The proposed method is an improvement over CSLBP. The CSLBP extracts the local pattern by thresholding the gray level intensity difference of center symmetric neighbouring pixels and ignores the center pixel in pattern calculation as shown in Fig1. The proposed method considers the original stimulus of intensity using differential excitation. Further, to produce compact pattern map the adjacent neighboring pixel pairs are considered for differential excitation computation and pattern map formation.

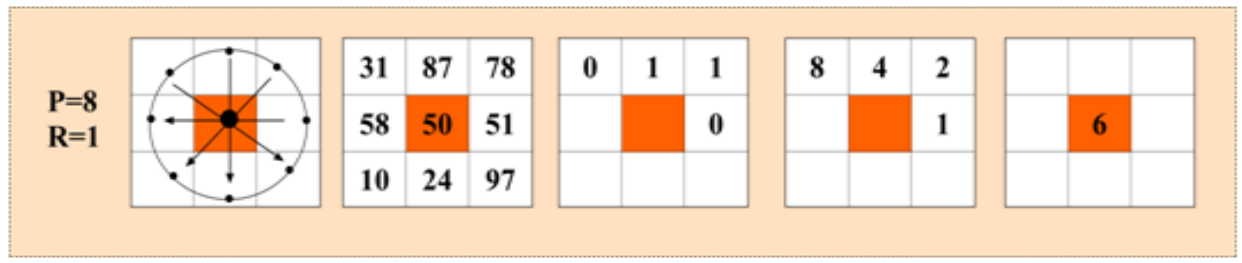

Fig1: Center symmetric local binary pattern (CSLBP)

\section{Feature extraction}

The proposed method extracts the local pattern in two steps. First, compute the differential excitation matrix for a given center pixel and second, encode the differential excitation matrix values using local binary pattern. The differential excitation matrix and the consideration of pixel candidates for obtaining the differential excitation matrix are shown in Fig $2 \mathrm{a}$ and $\mathrm{b}$ respectively. The example pattern map calculation is shown in Fig3.The computation of local differential excitation binary pattern is as follows

- First, compute the differential excitation values of the given center pixel at radius1 (i.e. $\mathrm{R}=1, \mathrm{P}=8$ ) as shown in Fig2. The four differential excitation values at radius 1 are obtained as follows

For $\xi_{1}$, consider the neighbourhood pixels candidates at $\left(0^{0}, 45^{0}\right)$ as shown in Fig $2 \mathrm{~b}$.

$$
\xi_{1}=\arctan \left[\frac{\left(Y_{c}-Y_{1}\right)}{Y_{c}}+\frac{\left(Y_{c}-Y_{2}\right)}{Y_{c}}\right]
$$

For $\xi_{2}$,consider pixel candidates in $90^{\circ}$ and $135^{\circ}$ directions at radius 1

$$
\xi_{2}=\arctan \left[\frac{\left(Y_{c}-Y_{3}\right)}{Y_{c}}+\frac{\left(Y_{c}-Y_{4}\right)}{Y_{c}}\right]
$$

For $\xi_{3}$,computation consider the pixel candidates along $180^{\circ}$ and $225^{\circ}$

$$
\xi_{3}=\arctan \left[\frac{\left(Y_{c}-Y_{5}\right)}{Y_{c}}+\frac{\left(Y_{c}-Y_{6}\right)}{Y_{c}}\right]
$$

For $\xi_{4}$,computation consider the pixel candidates along $270^{\circ}$ and $315^{\circ}$

$$
\xi_{4}=\arctan \left[\frac{\left(Y_{c}-Y_{7}\right)}{Y_{c}}+\frac{\left(Y_{c}-Y_{8}\right)}{Y_{c}}\right]
$$

- The obtained differential excitation values thus obtained are now encoded using LBP from equation (10)

$$
S\left(\xi_{i}\right)=\left\{\begin{array}{cc}
+1 & \xi_{i}>0 \\
0 & \xi_{i} \leq 0
\end{array} \quad \forall i=1,2,3,4\right.
$$

The pattern map values are obtained using equation 11 as

$$
\operatorname{PTN}\left(Y_{c}\right)=\left\{\begin{array}{c}
2^{0} \times S\left(\xi_{1}\right)+2^{1} \times S\left(\xi_{2}\right)+ \\
2^{2} \times S\left(\xi_{3}\right)+2^{3} \times S\left(\xi_{4}\right)
\end{array}\right.
$$

The proposed LDEBCoP obtains the pattern map values considering each image pixel as a center pixel in a given image and the pattern map is formed. In addition, gray level co-occurrence matrix(GLCM) is used to obtain the occurrence of pixels pairs in the pattern mapped image for better feature extraction. The intensity values in pattern mapped image vary from 0 to 15 . The length of GLCM matrix obtained on pattern mapped image is $16 \times 16$.

The proposed method obtains the occurrence of pixel pairs using GLCM at different directions and distances. In this paper, four combinations are used for co-occurrence of pixel pairs as shown in Fig4

- Combination\#1: Four GLCM of distance 1, and angles $0^{\circ}, 45^{\circ}, 90^{\circ}$ and $135^{\circ}$ have been obtained.

- Combination\#2: Four GLCM of distance 2, and angles $0^{\circ}, 45^{\circ}, 90^{\circ}$ and $135^{\circ}$ have been calculated. 


\begin{tabular}{|l|l|l|}
\hline$\xi_{4}$ & $\xi_{3}$ & $\xi_{2}$ \\
\hline & & $\xi_{1}$ \\
\hline & & \\
\hline
\end{tabular}

\section{Differential excitation matrix}

(a)

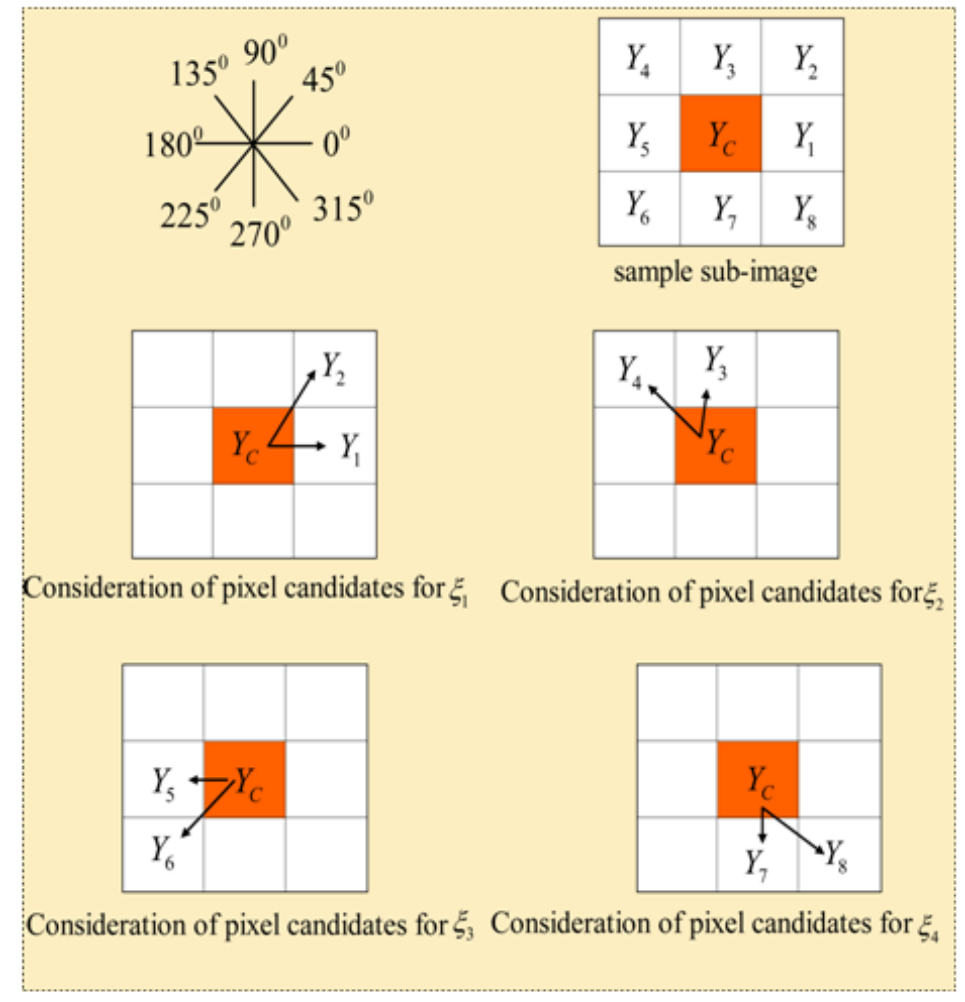

(b)

Fig2:a) Differential excitation matrix b)Consideration of pixel candidates to obtain differential excitation values

\begin{tabular}{|c|c|c|c|c|c|c|c|c|c|}
\hline \begin{tabular}{|l|l|l|}
118 & 113 & 103 \\
\end{tabular} & \begin{tabular}{|l|l|l|}
0.07 & -0.03 & -0.11 \\
\end{tabular} & 1 & 0 & 0 & 8 & 4 & 2 & & \\
\hline 10610689 & 0.18 & & & 1 & & & 1 & 9 & \\
\hline 110103101 & & & & & & & & & \\
\hline
\end{tabular}

Fig3: Example pattern map calculation

- Combination\#3: Two GLCM of distance 1 with angles $0^{\circ}, 45^{\circ}$ and two GLCM of distance 2 with angles $0^{0}, 45^{0}$ have been calculated

- Combination\#4: Two GLCM of distance 1 with angles $0^{\circ}, 90^{\circ}$ and two GLCM of distance 2 with angles $0^{0}, 90^{\circ}$ have been obtained.

Each GLCM has a length of $16 \times 16$ and each combination have four such GLCMs. Therefore, the length of feature vector is $4 \times 16 \times 16=1024$. 


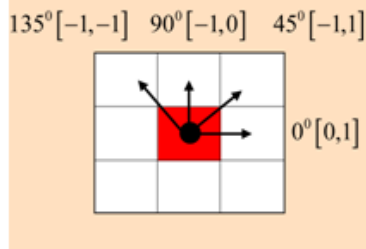

combination\#1

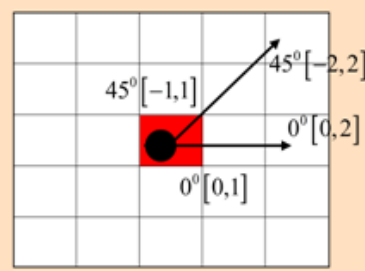

combination\#3

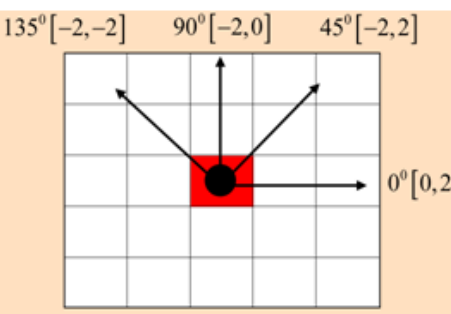

combination\#2

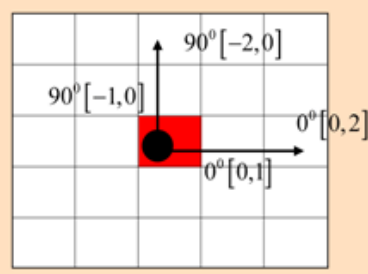

combination\#4

Fig4: Different combinations of GLCM

The algorithm to extract features from a given image is as follows

Input: Image

Output: Feature vector

Step1: Input the gray scale image or convert the image into gray scale if it is RGB image

Step2: Obtain differential excitation matrix using equations 6-10

Step3: Apply any one combination of GLCM as explained in the previous section at various distances and angles.

Step4: convert the four 16x16 matrices into vectors obtainedfrom previous step

Step5: concatenate all four vectors obtained in step4 into a single vector to form the feature vector of length of 1024.

\section{E. Similarity measure}

Similarity measure is used to retrieve $n$ top matches for the given query image from the feature database by measuring the distance between query image and image features in the database. Consider $f_{q}=\left(f_{1}, f_{2}, \ldots, f_{L}\right)$ be the query image feature vector of length $\mathrm{L}$ and $f_{d b i}=\left(f_{d b 1}, f_{d b 2}, \ldots, f_{d b N}\right)$ represents the feature vectors in the data base with $N$ number of images. The proposed method uses $d_{1}$ distance metric for similarity measure.

The $d_{1}$ is computed as follows

$$
d_{1}(q, q b)=\sum_{i=1}^{N}\left|\frac{f_{d b i}-f_{q}}{1+f_{d b i}+f_{q}}\right|
$$

Where $f_{d b i}$ is $i^{\text {th }}$ feature vector in the database, $f_{q}$ is query feature vector and $d_{1}(q, q b)$ is distance function.

\section{Experimental results}

The performance of the proposed method is evaluated based on the metrics, average precision rate (APR)and average recall rate(ARR).For $n$ number of images retrieved for each query image, the precision and recall are computed using equations 13 and 14 respectively

$$
p_{i}(n)=\frac{R_{V}}{R_{T}}
$$

Where $p_{i}(n)$ represents the precision for $i^{\text {th }}$ image in the database, $R_{V}$ gives the number of relevant images retrieved and $R_{T}$ is the total number of images retrieved. 


$$
r_{i}(n)=\frac{R_{v}}{R_{D}}
$$

Where $R_{D}$ is the total number of images in the database.

The average precision and recall for $j^{\text {th }}$ category with $N_{1}$ number of images are determined using the equations 15 and 16

$$
\begin{aligned}
& A P_{n}(j)=\frac{1}{N_{1}} \sum_{i=1}^{N_{1}} p_{i}(n) \\
& A R_{n}(j)=\frac{1}{N_{1}} \sum_{i=1}^{N_{1}} r_{i}(n)
\end{aligned}
$$

The average precision rate (APR) and average recall rate(ARR) for a given database with $N_{2}$ categories are obtained using the formulae

$$
\begin{aligned}
& A P R=\frac{1}{N_{2}} \sum_{i=1}^{N_{2}} A P_{n}(i) \\
& A R R=\frac{1}{N_{2}} \sum_{i=1}^{N_{2}} A R_{n}(i)
\end{aligned}
$$

\section{F. Experiment\#1}

The database for experiment\#1 is KTH-TIPS texture database [29]. The KTH-TIPS database consists of texture images of ten materials under varying illumination, scale and pose. The materials are Sand paper, Styrofoam, Crumpled aluminum foil, Sponge, Corduroy ,Linen, Cotton, Orange peel , Brown bread and Cracker B. The database consists of 10 categories each category consists of 81 images of same material taken at different illumination, scale and pose.Therefore, $81 \times 10=810$ texture images of size $200 \times 200$ are present in the database. The performance of the proposed method in comparison with LTCoP and CSLBCoP in terms of average precision rate (APR) and average recall rate (ARR) on KTH-TIPS texture database is depicted in Figs 5 and 6.The performance of proposed method with other existing methods on gray scale texture images are detailed below

- The APR of LDEBCoP has improved from $65.65 \%$ and $68.61 \%$ to $73.95 \%$ as compared with LTCoP and CSLBCoP respectively on KTH-TIPS texture database for the top 20 images retrieved as shown in Fig5.

- The ARR has significantly improved from $40.46 \%$ and $43.68 \%$ to $46.22 \%(n=100)$.

The experimental results clearly show that the proposed LDEBCoP performed superior to LTCoP and CSLBCoP.

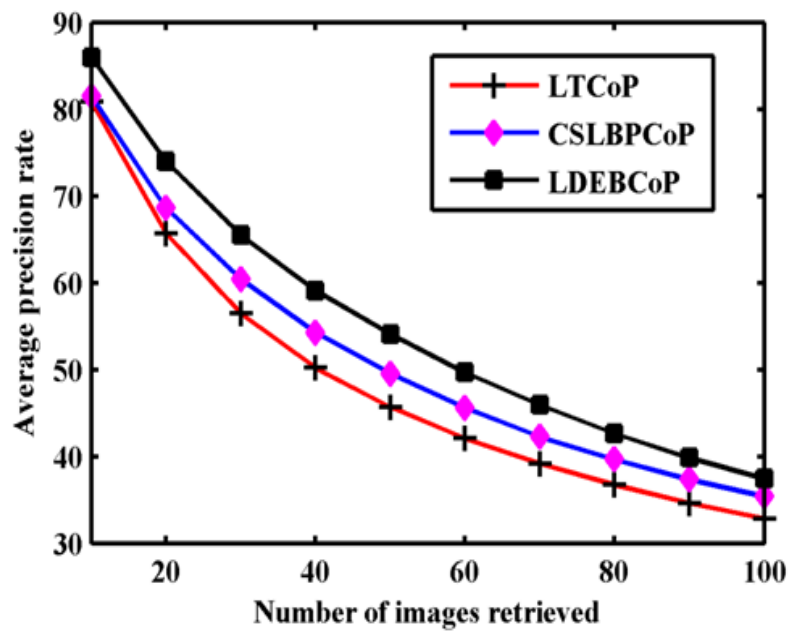

(a) 


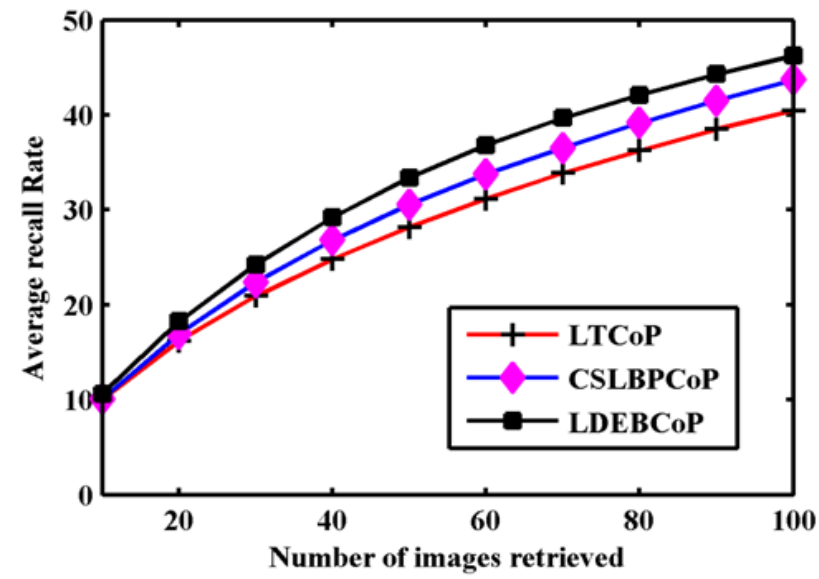

(b)

Fig5: APR and ARR curves for KTH-TIPS texture database

\section{G. Experiment\#2}

The Outex_TC_00010 test database [30] is used for evaluating the performance of the proposed method. The test database consists of 4320 texture images with 24 classes of textures. The images are collected with illumination "Inca" at a resolution of 100dpi and nine different rotation angles viz 0, 5, 10, 15, 30, 45, 60, 75, and 90. Each class consists of 20 non-overlapping texture images of size $128 \times 128$. Hence total images are $24 \times 20 \times 9=4320$. The performance of proposed LDEBCoP in comparison with LTCoP and CSLBCoP in terms of average precision is depicted in Fig7. The retrieval results of LDEBCoP along with other existing methods in terms of ARR on OUTEX database illustrated in Fig8. The experimental results indicates that the LDEBCoP method's average precision (71.26\%) greater than LTCoP (67.21\%) and CSLBCoP (69\%) for the top 100 retrieved images on OUTEX texture database. It is also observed that the LDEBCoP average recall (39.5\%) is greater than LTCoP (37.3\%) and CSLBCoP (38\%).

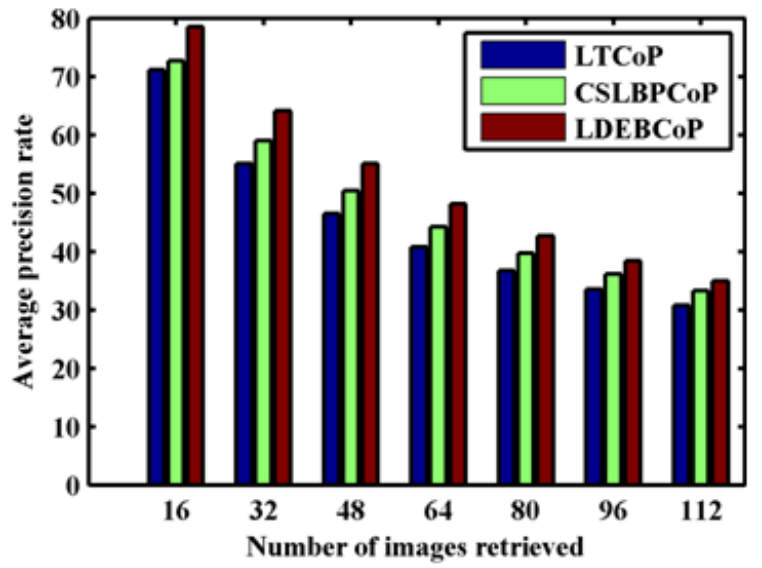

(a)

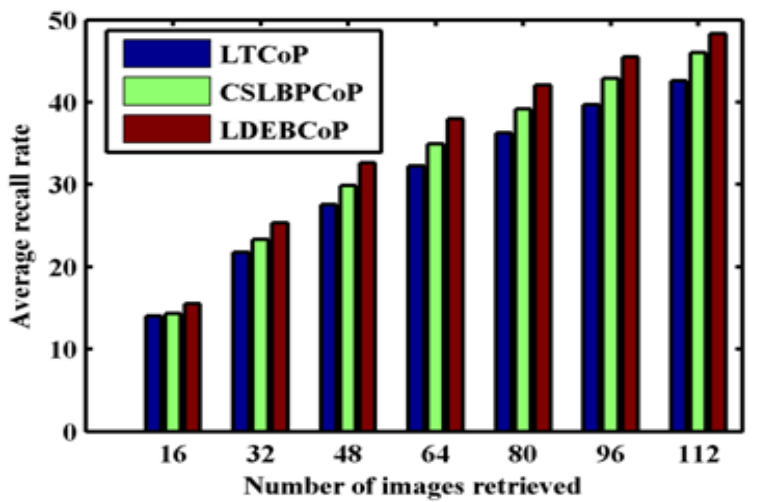

(b)

Fig6: Average precision and recall rates for KTH-TIPS texture database. 


\section{H. Experiment\#3}

Computer tomography (CT) database for performance evaluation of different computer aided detection systems is created by Vision and Image analysis (VIA) group and International early lung cancer action program (I-ELCAP) [31]. The images are presented in DICOM (digital imaging and communications in medicine) format which are obtained in a single breadth hold with a $1.25 \mathrm{~mm}$ slice thickness. For the experiment $10 \mathrm{CT}$ scans are considered and each scan has 100 images with resolution $512 \times 512$. Hence total images are $10 \times 100=1000$

For performance evaluation, average precision rate has been calculated for proposed LDEBCoP and other existing methods. In Fig9, average precision with number of images retrieved has been plotted. The LDEBCoP precision $(84.15 \%)$ is greater than LTCoP $(82.08 \%)$ and CSLBCoP $(78.94 \%)$. The retrieval results are depicted in Fig10. The ARR of LDEBCoP (50.68\%) outperformed LTCoP (48.9\%) and CSLBCoP $(47.09 \%)$. The experimental results indicate that the proposed method outperformed other existing methods.

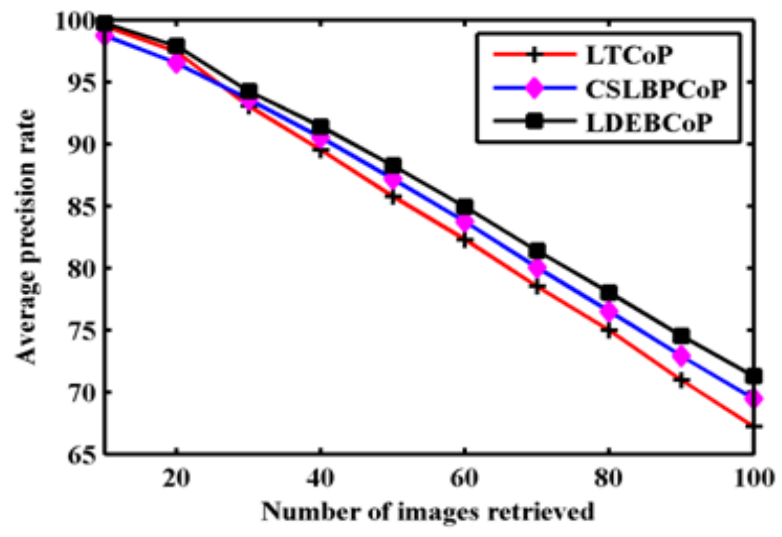

(a)

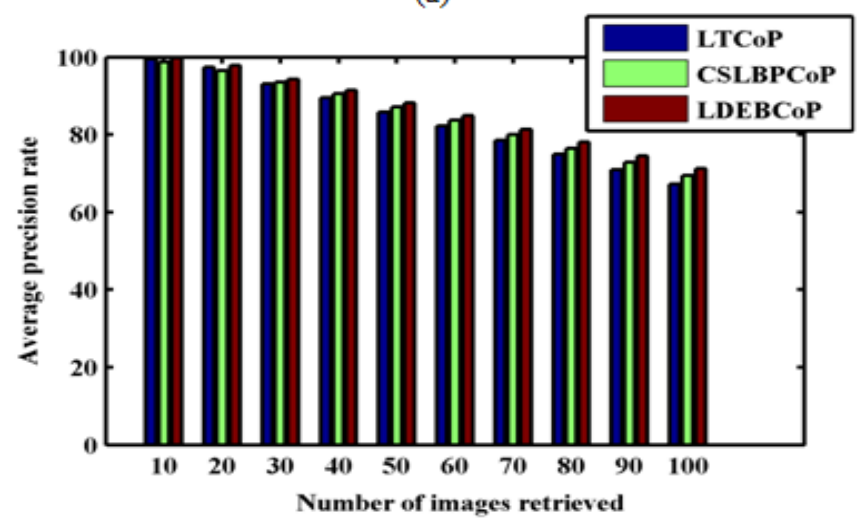

(b)

Fig7: Average precision rate for OUTEX texture database

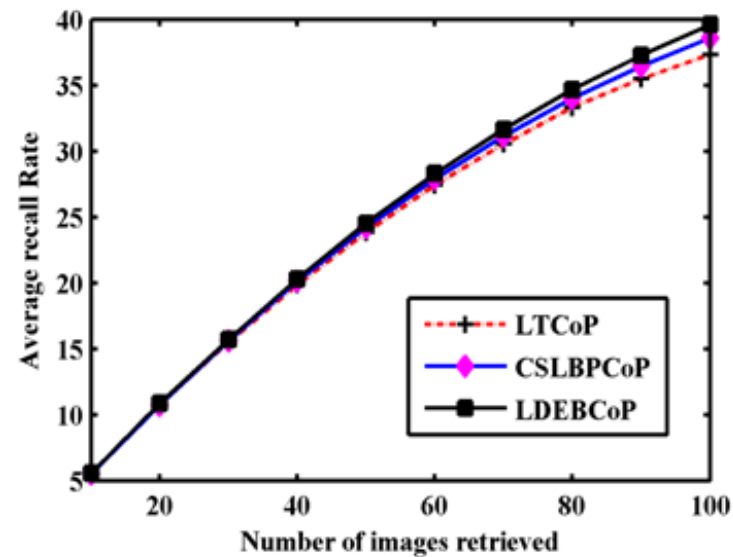

(a) 


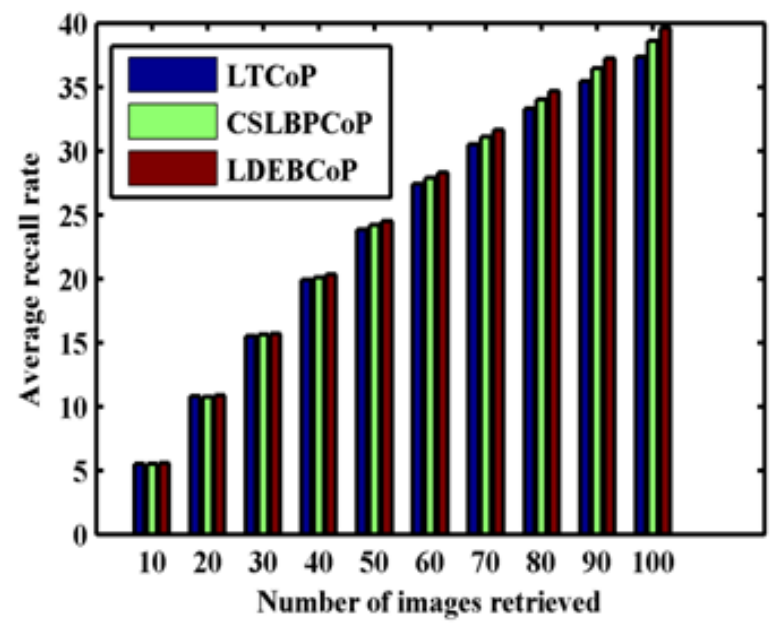

(b)

Fig8: Recall performance of OUTEX texture database

\section{Experiment\#4}

The National Electrical Manufacturers Association (NEMA) created a standard, named as DICOM for the distribution and viewing of medical images such as CT scans and ultra sound scans etc [32]. For the experiment\#4 , the database formed by 797 collected CT scans of different parts of human body and these are group into 10 categories $(104,37,128,54,48,75,37,131,54,129$ images). The performance measurement, APR and ARR has been calculated for each method, including the proposed LDEBCoP as shown in Figs $11 \mathrm{a}$ and $\mathrm{b}$. The experimental results indicates that the performance of proposed method is superior compared with other existing methods. The proposed LDEBCoP performance in terms of APR on different databases is shown in Table1.

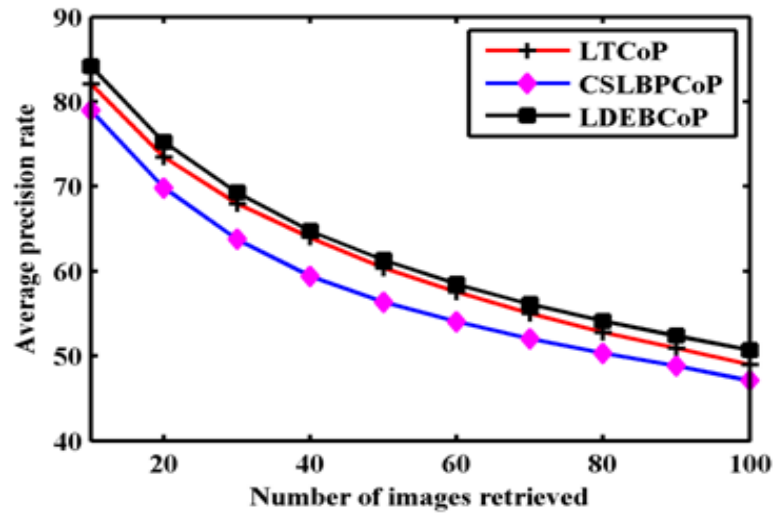

(a)

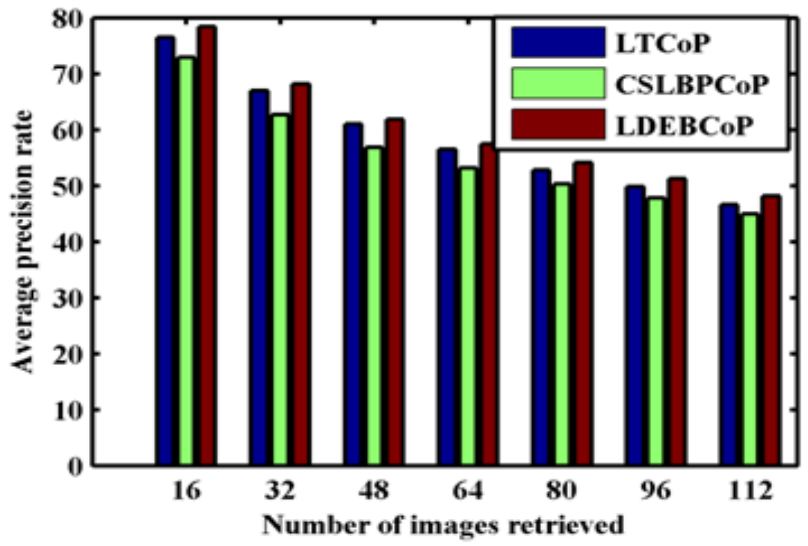

(b)

Fig:9 Average precision rate for VIA/I- ELCAP data base

Table1 LDEBCOP method with different direction and distance in GLCM 


\begin{tabular}{|c|c|c|c|c|}
\hline $\begin{array}{c}\text { GLCM } \\
\text { Combination } \\
\#\end{array}$ & $\begin{array}{c}\text { KTH-TIPS } \\
\text { APR }(\%)\end{array}$ & $\begin{array}{c}\text { OUTEX } \\
\text { database } \\
\text { APR }(\%)\end{array}$ & $\begin{array}{c}\text { VIA/I- } \\
\text { ELCAP } \\
\text { APR }(\%)\end{array}$ & $\begin{array}{c}\text { NEMA- } \\
\text { CT } \\
\text { APR }(\%)\end{array}$ \\
\hline 1 & $\mathbf{8 5 . 9 5}$ & 99.34 & 80.86 & 74.7 \\
\hline 2 & 84.43 & $\mathbf{9 9 . 7 1}$ & $\mathbf{8 4 . 1 5}$ & $\mathbf{7 9 . 9 6}$ \\
\hline 3 & 85.20 & 99.54 & 82.64 & 76.44 \\
\hline 4 & 85.46 & 99.50 & 81.79 & 75.82 \\
\hline
\end{tabular}

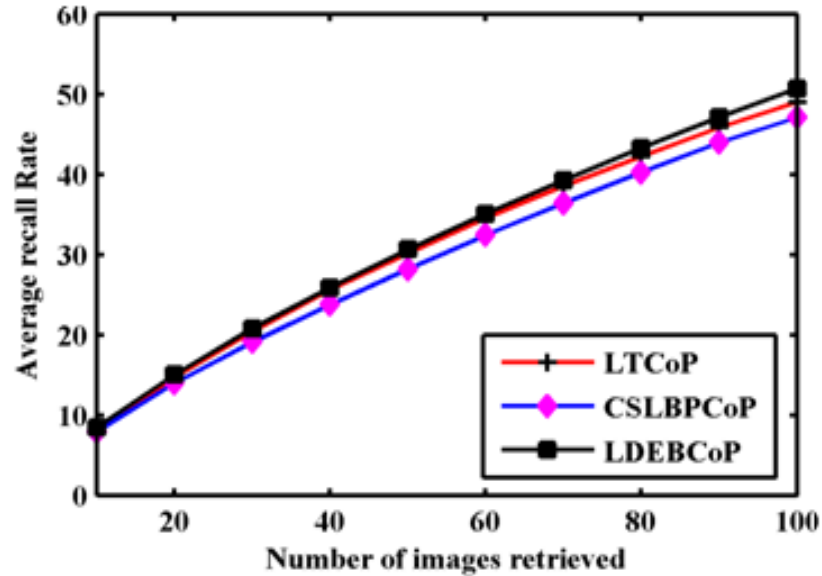

(a)

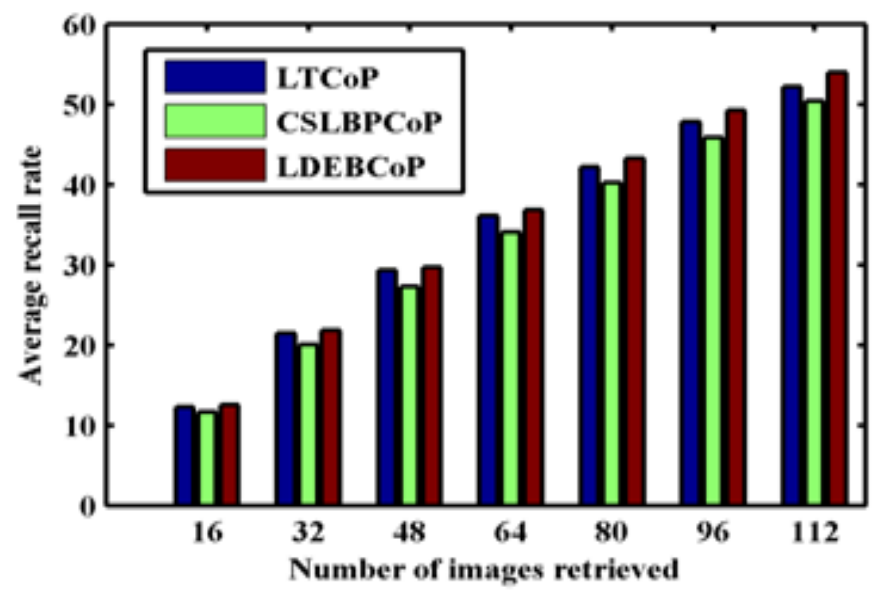

(b)

Fig10: Retrieval performance of LDEBCoP with other existing methods on VIA/I- ELCAP data base

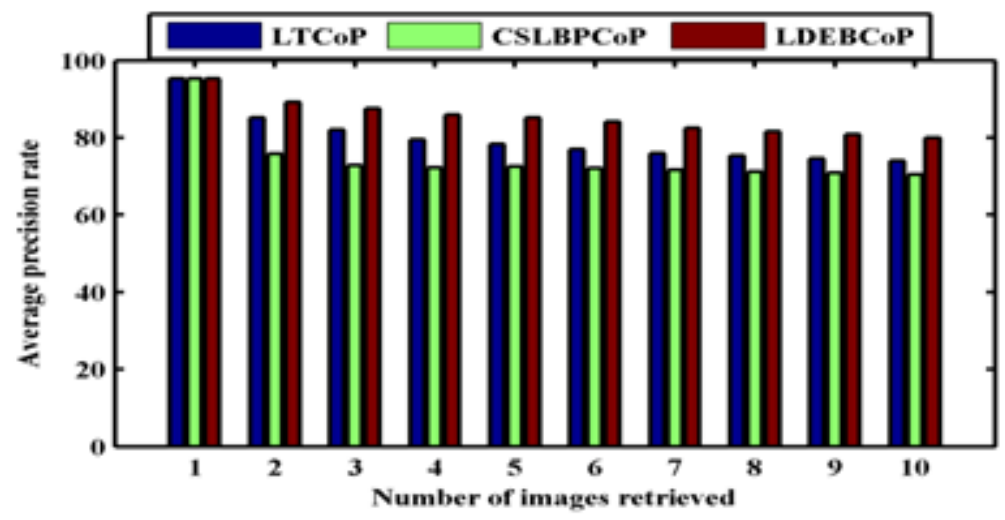

(a) 


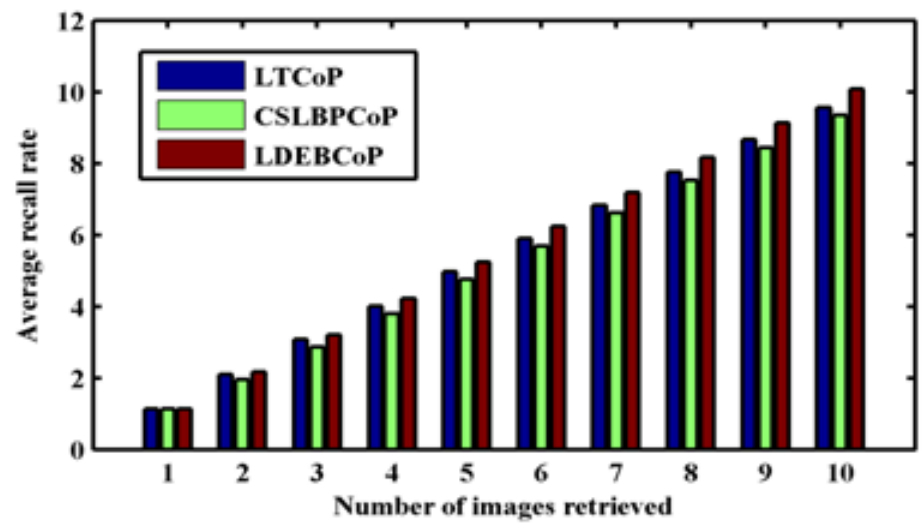

(b)

Fig11: Average precision and recall rates for NEMA-CT medical image database

\section{Conclusions}

In the proposed work, a new feature descriptor named LDEBCoP has been developed for texture and bio-medical image retrieval. This method extracts the local structure information using the differential excitation, to simulate human being's perception of patterns. Unlike CSLBP, the proposed method extracts local structure information using adjacent neighbourhood pixel pairs which are more related compared to center symmetric neighbourhood pixel pairs. Further, the mutual co-occurrences of pattern map values are obtained using GLCM, which is robust compared to the histogram technique. Experimental results clearly show that LDEBCoP illustrates a favourable performance on four databases viz ., KTH-TIPS, OUTEX texture database,NEMA-CT and VIA/I- ELCAP database. Specifically, the LDEBCoP achieved ARR of $46.22 \%$ compared LTCoP (40.46\%) and CSLBCoP (43.68) and 39.5\% on OUTEX database compared to LTCoP (37.3\%) and CSLBCoP (38\%). The experimental results also demonstrated the effectiveness of proposed method on NEMA-CT and VIA/I- ELCAP bio medical image database.

\section{References}

[1] Ahonen, T., Hadid, A., and Pietikäinen, M., 2004, "Face recognition with local binary patterns", Computer Vision-ECCV 2004( Springer)., pp. 469-481.

[2] Ning,J., Zhang,D., and Wu,C.,2009 , "Robust object tracking using joint color texture histogram”, J. Pattern Recognit. Artif. Intell.,23(7), pp. 1245-1263.

[3] Pietikäinen,M.,and Mäenpää,T.,2005, Texture analysis with local binary patterns, The Handbook of Pattern Recognition and Computer Vision, World Scientific Publishing, Singapore, pp. 197-216.

[4] Fernandez,A., Ghita, O., Gonzalez, E., Bianconi,F., and Whelan, P.F.,2011, "Evaluation of robustness against rotation of LBP, CCR and ILBP features in granite texture classification”, Trans.on Mach. Graph. Vision., 22(6), pp. 913-926.

[5] Pietikäinen,M., Hadid, A.,Zhao,G., and Ahonen, T.,2011, Computer vision using local binary patterns, Computational Imaging and Vision(springer),London. doi:10.1007/978-0-85729-748-8.

[6] Choi, J.Y., Ro, M.Y., and Plataniotis, N.K., 2012, "Color local texture features for color face recognition", IEEE Trans. Image Process., 21(3), pp. 1366-1380.

[7] Huang,D., .Shan, C., Ardabilian, M., Wang, Y., and Chen, L.,2011, "Local binary patterns and its application to facial image analysis: a survey”, IEEE Trans. Syst. Man Cybern., 41(6),pp.765-781.

[8] Ojala, T., Pietikäinen,M., and Harwood, D.,1996, "A comparative study of texture measures with classification based on feature distributions", Trans.pattern recogn., 29(3),pp. 51-59.

[9] Zhao,Y.,2012, Theories and applications of LBP: a survey, Advanced Intelligent Computing Theories and Applications with Aspects of Artificial Intelligence, Lecture Notes in Computer Science, vol. 6839, pp. 112-120. Springer, Berlin.

[10] Guo, Z., Zhang,L., and Zhang, D.,2010, "Rotation invariant texture classification using LBP variance (LBPV) with global matching", Trans. pattern recognition, 43(3),pp. 706-719.

[11] Ojala, T., Pietikäinen,M., and Mäenpää,T.,2002, "Multiresolution gray scale and rotation invariant texture classification with local binary patterns", IEEE Trans. Pattern Anal. Mach. Intel., 24(7),pp. 971-987.

[12] Liao,H.W., 2010, "Region description using extended local ternary patterns", Proceedings of the 20th IEEE International Conferenceon Pattern Recognition (ICPR'10), Istambul, Turkey,pp. 1003-1006

[13] Pan, H., Xia,S.Y.,Jin,Z.L., and Xia,Z.L.,2011, "Illumination invariant face recognition based on improved local binary pattern", Proceedings of the 30th Chinese Control Conference (CCC'2011), Yantai, China, pp. 3268-3272.

[14] Tan, X., and Triggs,B.,2010, "Enhanced local texture feature sets for face recognition under difficult lighting conditions", IEEE Trans. Image Process. , 19(6), pp. 1635-1650.

[15] Mäenpää,T., and Pietikäinen,M.,2004, "Classification with color and texture: jointly or separately", Trans.Pattern Recogn., 37, pp.1629-1640.

[16] Manjunath,N.K., Renuka,A., and Niranjan, U.C.,2007, "Linear models of cumulative distribution function for content-based medical image retrieval", J. Med. Syst. ,31,pp.433-443.

[17] Seng, W.C., and Mirisaee,H.S., "Evaluation of a content based retrieval system for blood cell images with automated methods", J. Med. Syst., doi: 10.1007/s10916-009-9393-3. 
[18] Zakeri, F. S., Behnam, H., and Nasrin,A.,2010, "Classification of benign Malignant breast masses based on shape and texture features in sonography images", J.Med.Syst.,36,pp.1621-627.

[19] Traina, A., Castanon, C., and Traina, C Jr.,2003 "Multiwave med: A system for medical image retrieval through wavelets transformations",Proc. 16th IEEE Symp. Comput.-Based Med. Syst., New York,USA, pp.150-155.

[20] Felipe, J. C., Traina, A. J. M., and Traina, C. Jr.,2003, "Retrieval by content of medical images using texture for tissue identification", 16th IEEE Symp. Comput.-BasedMed. Syst., New York, USA, pp.175-180.

[21] Müller, H., Rosset, A., Vallét, J. P., and Geisbuhler, A., 2004,"Comparingfeature sets for content-based image retrieval in a medical case database", Proc. SPIE Med. Imag., PACS Imag. Inf., San Diego,USA, 99-109.

[22] Murala,S., Maheshwari,P.R., and Balasubramanian,R.,2012, , "Directional binary wavelet patterns for biomedical image indexing and retrieval", J. Med. Syst., 36(5), pp. 2865-2879.

[23] Murala,S., and Wu,J.,2013, Local mesh patterns versus local binary patterns: biomedical image indexing and retrieval", IEEE J. Biomed. Health Infor., 18(3), pp. 929-938.

[24] Zhang, B., Gao, Y., Zhao, S., and Liu, J.,2010, "Local derivative pattern versus local binary pattern: face recognition with highorder local pattern descriptor", IEEE Trans. Image Process, 19(2),pp.533-44.

[25] Manisha, Verma., and Raman, B.,2015, "Center symmetric local binary co-occurrence pattern for texture, face and bio-medical image retrieval”, J. Vis. Commun. Image Represent.,32, pp. 224-36.

[26] Subrahmanyam, M. , and Wu, Q.J. , 2013 ,"Local ternary co-occurrence patterns: a new feature descriptor for MRI and CT image retrieval", Neuro computing., 119(6),pp. 399-412.

[27] Heikkilä, M., Pietikäinen, M. , and Schmid, C.,2006, "Description of interest regions with center-symmetric local binary patterns", Trans. Computer Vision, Graphics and Image Processing., pp. 58-69.

[28] Chen, J., Shan, S., and Zhao, G., 2009, "A robust descriptor based on Weber's Law”, IEEE Trans. Pattern ana.Mach.Intell., 32(.9),pp 1705-1720.

[29] KTH-TIPS texture database. Available: http://www.nada.kth.se/cvap/databases/kth-ips/download.html.

[30] Outex texture database. Available: http://www.outex.oulu.fi/index.php?page=retrieval.

[31] VIA/I-ELCAP CT lung image dataset, Available[online] http://www.via.cornell.edu/-databases/lungdb.html

[32] NEMA-CT imagedatabase. Availablefrom[Online]: 〈ftp://medical.nema.org/ medical/Dicom/Multiframe/ $\rangle$.

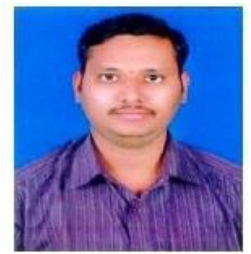

G V SATYA KUMAR obtained his B.Tech degree from JNT University, Hyderabad in year 2002 and M.Tech degree from ANU, Guntur in the year 2008. Presently he is pursuing $\mathrm{Ph}$. D in image processing under the guidance of P.G. Krishna Mohan. His areas of interests are image retrieval, object tracking

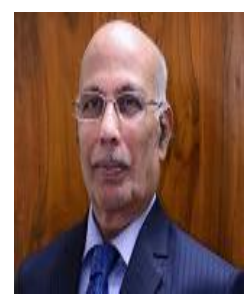

P G Krishna Mohan was born in India in1952. He received the Ph.D degree in signal processing from Indian Institute of Science, Bangalore, India in 1988. He is currently working as Professor in Institute of Aeronautical Engineering, Hyderabad. He Worked as Head of ECE Dept., JNTUCEH, Member of BOS for ECE faculty at University Level, Chairman of BOS of EIE group at University level, Chairman of BOS of ECE faculty for JNTUCEH, Member of selection committees for Kakitiya, Nagarjuna University, DRDL and convener for Universities Hidian committees. He has more than 50 papers in various International and National Journals and Conferences. His areas of specialization are Signal Processing, Signal Estimation, Probability Random Variables and Communications. 